\title{
L'organisation des connaissances dans les thésaurus : une approche pragmatique cognitive
}

The Organization of Knowledge in a Thesaurus : A Cognitive Pragmatics

Approach

\section{El-Khansa Mkada}

\section{(2) OpenEdition Journals}

Édition électronique

URL : http://journals.openedition.org/edc/4030

DOI : $10.4000 /$ edc. 4030

ISSN : 2101-0366

Éditeur

Université Lille-3

Édition imprimée

Date de publication : 1 décembre 2012

Pagination : $139-154$

ISBN : 978-2-917562-08-6

ISSN : $1270-6841$

Référence électronique

El-Khansa Mkada, «L'organisation des connaissances dans les thésaurus : une approche pragmatique cognitive », Études de communication [En ligne], 39 | 2012, mis en ligne le 01 décembre 2012, consulté le 03 mai 2019. URL : http://journals.openedition.org/edc/4030 ; DOI : 10.4000/edc.4030

Ce document a été généré automatiquement le 3 mai 2019.

(c) Tous droits réservés 


\title{
L'organisation des connaissances dans les thésaurus : une approche pragmatique cognitive
}

\author{
The Organization of Knowledge in a Thesaurus : A Cognitive Pragmatics \\ Approach
}

El-Khansa Mkada

\section{Introduction}

1 La pragmatique cognitive est une théorie élaborée par l'anthropologue Dan Sperber, en collaboration avec la linguiste Deirdre Wilson (Sperber et Wilson, 1989). Elle a pour objet l'étude du processus communicationnel et l'interprétation des énoncés dans le cadre d'une relation interactive entre un locuteur et un destinataire, où le sens est inféré par ce dernier à partir de ce que le locuteur lui dit et du contexte. Ses fondements reposent sur la théorie des actes de langage (Austin, 1970), dont notamment les principes de Grice (1957), actualisés à la lumière du cadre théorique de la modularité de l'esprit proposé par Fodor (1986). La conjugaison de ces théories a mis en évidence la modularité du langage, tout en accentuant la distinction entre les processus inférentiels et les codes linguistiques. L'approche de cette théorie se présente ainsi :

Qui adopte une perspective pragmatique est amené à concevoir les énoncés non tant comme des moyens qu'utilise le locuteur pour transmettre au moyen de sons le sens qu'il veut communiquer, mais plutôt comme des indices riches et complexes que le locuteur fournit à l'auditeur afin de lui permettre de reconstruire le sens voulu (Sperber et Origgi, 2005, 236).

Nous présentons les principales notions de la pragmatique cognitive avant de les adapter à l'étude des thésaurus tout en tenant compte de ses spécificités, dont le fait d'être un écrit n'est pas la moindre. Ces spécificités vont servir la mise en place progressive des principes de base du type d'approche pragmatique que nous préconisons pour l'étude de notre objet. 


\section{La modularité}

\section{Modularité du langage}

3 La pragmatique cognitive a appliqué à l'étude du langage la théorie de la modularité selon laquelle l'esprit humain est composé de plusieurs modules innés et spécialisés qui fonctionnent automatiquement, inconsciemment, très rapidement, en parallèle et indépendamment les uns des autres. Chaque module est décomposable en sous-modules destinés à traiter un type très limité d'informations en provenance du monde extérieur, et un système central est chargé d'intégrer les différentes opérations entre elles et permet au cerveau de coordonner les différents modules.

4 Sperber et Wilson (1989) expliquent que le traitement des composants des messages verbaux relève du système périphérique qui fournit la forme logique de l'énoncé au système central, lequel effectue une analyse conjointe des données contextuelles pour les interpréter.

5 La forme logique se caractérise par une suite structurée de concepts renvoyant chacun à une adresse dans la mémoire à long terme, et fournissant des informations, ou prémisses, de trois types :

- logique : qui correspond aux relations logiques qu'un concept peut entretenir avec d'autres concepts;

- encyclopédique: qui regroupe toutes les connaissances qu'a le sujet sur les objets qui tombent, ou qui sont censés tomber, sous le concept;

- lexical : qui correspond aux contreparties en langue(s) naturelle(s) du concept.

6 Les données contextuelles, entendues comme un ensemble d'assomptions ou de propositions, proviennent de trois sources :

- l'interprétation des énoncés immédiatement précédents, conservés dans la mémoire à moyen terme, pour servir l'interprétation de l'énoncé en cours de traitement ;

- l'environnement physique, tel que les caractéristiques du lieu dans lequel se situe la communication ;

- la mémoire à long terme, dans laquelle se trouvent des informations de nature diverse sur le monde et dont certaines pourront être intégrées au contexte.

7 La vision modulaire du langage place donc la linguistique au niveau du module périphérique dont le siège est la mémoire à court terme. Celle-ci explicite le niveau codique en relation avec l'ensemble des processus inférentiels au niveau du module central dont le siège est la mémoire à long terme.

\section{Modularité des thésaurus}

8 Les thésaurus contiennent une introduction, un sommaire thématique, une liste thématique, une liste alphabétique, une liste hiérarchique et un index permuté. Dans les thésaurus multilingues s'ajoutent des équivalents linguistiques. 


\section{L'introduction}

9 L'introduction est consacrée à l'objectif du thésaurus, à la présentation de l'institution qui l'a conçu, à l'explication de sa structure et de son usage, au mode de mise à jour et enfin à la citation des documents-sources, utilisés ou créés par l'institution, dans lesquels elle a puisé les termes de son langage.

10 L'organisation de la connaissance et les représentations conceptuelles sont prises en charge par le concepteur du thésaurus qui s'avère être un spécialiste en son domaine et au service d'une institution. Les destinataires du thésaurus sont désignés : il s'agit d'abord des indexeurs, afin d'assurer une meilleure harmonisation dans le traitement de l'information, et ensuite des utilisateurs des systèmes d'information.

11 A ce stade, nous pouvons déjà ajouter un type de prémisses fonctionnelles et utilitaires dans le cadre de la forme logique des énoncés préconisée par la pragmatique cognitive. Les informations contenues dans l'introduction regroupent toutes les connaissances de type encyclopédique permettant de déterminer l'objet «thésaurus ». Elles sont intégrées dans la mémoire à long terme des destinataires qui ont une représentation complète de cet objet.

\section{Le sommaire thématique}

Le sommaire thématique présente la liste des facettes correspondant aux préoccupations de l'institution. Ce sont les seuls éléments non répétitifs du thésaurus, inappropriés pour l'indexation, et pouvant être perçus comme autant de modules car c'est à travers ces facettes que l'indexeur peut accéder aux sous-modules. Par exemple ${ }^{1}$ :

QUESTIONS JURIDIQUES ET POLITIQUES

SCIENCES ET TECHNIQUES

Par un effet de primauté, la mémoire à court terme focalise sur ces éléments hiérarchisés, écrits en majuscules et remarquables par leur stabilité.

\section{La liste thématique}

La liste thématique regroupe tous les descripteurs au sein de la facette qui encapsule le thème en indiquant les termes rejetés figurant toujours en caractères italiques. Chaque descripteur est précédé d'une indication sur son statut, comme par exemple :

[Facette] QUESTIONS POLITIQUES ET JURIDIQUES

[Thème] DÉSARMEMENT ET QUESTIONS MILITAIRES

[Descripteur] ABRIS ANTI-BOMBES

[Non-descripteur] ABRIS ANTI-RAIDS-AÉRIENS

La compréhension de la facette n'est pas la somme de celle des termes qu'elle recouvre; elle semble servir uniquement de point d'ancrage conceptuel. Pour le concepteur d'un système de signes non phrastique, la modularité semble le moyen d'inférer le sens des termes qu'il emploie, non à travers le niveau codique du descripteur mais à travers le contexte dans lequel il le situe, de sorte que le lecteur intègre les inférences mentalement en même temps que le code qui lui est associé. Si le concepteur ne peut agir directement sur le sens inhérent aux concepts, il peut le déterminer en traçant un passage obligé vers leur signification par le point de vue de l'institution. 


\section{La liste alphabétique}

16 La liste alphabétique reclasse tous les descripteurs, indépendamment de leur facette d'appartenance, et les fait apparaître en caractères gras suivis par leur champ sémantique, comme par exemple :

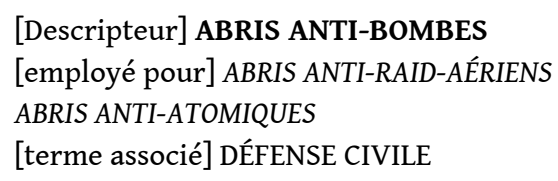

17 Tout se passe comme si les facultés cognitives du concepteur ont fonctionné dans l'objectif d'épargner à l'indexeur d'utiliser les siennes. Les multiples entrées composant la liste alphabétique constituent des représentations implémentées dans un paradigme qui rend la reconnaissance du descripteur recherché quasiment dépendante du guidage programmé de sa perception.

\section{La liste hiérarchique}

La liste hiérarchique répertorie les descripteurs génériques, en caractères gras, suivis de leurs spécifiques, en mettant en évidence les degrés de profondeur au moyen d'un décalage accentué par des points :

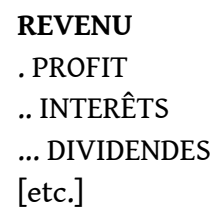

19 Cette structuration se présente de telle sorte que l'hémisphère droit, siège de la perception, soit sollicité plus souvent, et même avant l'hémisphère gauche. L'attention est en effet d'abord captée par la configuration de l'espace et par la graphie, puis elle se focalise sur les descripteurs.

\section{L'index permuté}

L'index permuté reprend la totalité des descripteurs en les réorganisant par ordre alphabétique en fonction des mots significatifs qui les constituent. Un descripteur apparaitra ainsi dans l'index autant de fois qu'il comporte de mots, dans l'ordre alphabétique de ces mots :

\section{ASSURANCE DE CRÉDIT AGRICOLE \\ CARTES DE CRÉDIT \\ CRÉDIT \\ CRÉDIT À LA CONSOMMATION \\ [etc.]}

21 Dans ce contexte, l'indexeur est constamment en interaction avec les connexions paradigmatiques établies entre les descripteurs, ce qui favorise intuitivement la perception des ensembles.

\section{Les équivalents dans d'autres langues}

Le thésaurus multilingue met à disposition un lexique des descripteurs traduits de la langue source vers des langues cibles. 
Les thésaurus peuvent être perçus comme un ensemble complexe de modules parallèles contenant invariablement les mêmes descripteurs dont l'organisation reflète les constructions cognitives de fragments du monde par l'institution conceptrice, et dont la réorganisation serait une façon de la réitérer.

4 Nous adhérons donc à la pragmatique cognitive pour qui le processus de la communication inter-individuelle non verbale ne peut être expliqué en dehors des notions d'ostentation et d'inférence, et qui y participent de la manière suivante dans les thésaurus :

- les stimuli visuels relevant de l'ostentation sont produits par le communicateur et seraient les indications qui précèdent chaque terme du thésaurus pour en préciser le statut (descripteur, terme associé, terme spécifique, etc.);

- les styles d'écriture et l'organisation paradigmatique des entrées participent de l'inférence, stimulent l'élimination des termes écrits en italique ainsi que les facettes, permettent de focaliser sur le descripteur et demandent l'effort de traitement le plus faible.

Nous estimons cependant que la structure du thésaurus porte le reflet de la gestalt (théorie de la forme, du verbe gestalten - donner une structure signifiante), théorie charnière entre behaviorisme et cognitivisme. En fait, la structuration des différentes listes cible l'hémisphère droit, siège de la perception, mais leur contenu cible aussi dans le même temps l'hémisphère gauche, siège de l'abstraction. Les percepts descripteurs sont ainsi constamment incrémentés par les stimuli visuels fournis par la structure. La connexion entre les deux hémisphères a pour effet d'abréger le séjour des descripteurs dans la mémoire sensorielle et tend à les inscrire d'emblée dans la mémoire de travail dont la fonction est de filtrer en quelques fractions de secondes des milliers de descripteurs, et ce peu importe les conditions dans lesquelles nous nous trouvons, car un indexeur ne travaille pas dans l'isolement. La structure de ces listes rend plus effi ciente la perception sélective des descripteurs en tant que tels, et par la suite celle des descripteurs adéquats. Le descripteur comporte d'abord une information sur sa qualité intrinsèque de descripteur.

Plus précisément, sur le plan visuel, c'est l'insight (découverte par intuition) qui repose sur le passage souvent brutal d'un état à un autre état, ou d'une liste à une autre, qui provoque la prise de conscience d'une restructuration de la perception d'une situation et dont l'effet est l'inhibition d'un comportement à la faveur de la répétition et de la diversité des combinaisons d'une masse de stimuli. Les gestaltistes soulignent d'ailleurs que l'information est perçue en fonction de ce que l'on connaît déjà et qui se trouve dans la mémoire à long terme. Dans les thésaurus, cette connaissance préalable est assurée par le fait que l'indexeur sait dans quel environnement il fonctionne et qu'il connaît la structure des thésaurus.

Lors de la recherche d'un descripteur, l'insight procure un gain de temps sur les plans de son repérage et de son transfert vers la mémoire de travail à long terme dont la capacité limitée, sur le plan de l'effort de mémorisation, se trouve dopée grâce aux restructurations. Ce gain se répercute dans les deux mémoires à long terme, l'épisodique et la procédurale. La première conservera l'ensemble des indices nécessaires à la récupération des descripteurs, alors que la seconde amplifiera l'habileté acquise dans la manipulation des listes.

On note que la modularité est une notion mouvante car un descripteur peut être spécifique par rapport à celui qui le précède et générique par rapport à celui qui le suit. 
On peut aussi capter certaines fissures dans le cloisonnement des modules (les facettes) sous l'effet des termes associés relevant d'autres modules. En permettant la circulation inter-modulaire des descripteurs, ces fissures cimentent la structure globale et la maintiennent, en conférant du même coup sa cohérence à l'ensemble de l'organisation de la connaissance.

\section{Les actes de langage}

\section{Les énonciations performatives}

29 Les énonciations performatives définies par Austin (1970) sont à l'origine de la théorie des actes de langage. Elles ne sont ni vraies ni fausses car elles sont l'acte en lui-même (le oui [je le veux] prononcé lors de la cérémonie du mariage en est un exemple). Grice (1957) a étendu cette théorie à l'intention de communication dont la linguistique, limitée au niveau codique, ne peut rendre compte.

Pour la pragmatique cognitive, les codes sont perçus par le module périphérique qui les transmet au module central de la pensée. Ainsi, la notion de code désigne « un ensemble d'associations entre, à chaque fois, un message et un signal » (Sperber, 2000, 120).

Quant à l'inférence, c'est un " processus qui part de prémisses et aboutit à une conclusion, qui en ce sens fonctionne comme un raisonnement, mais qui peut opérer de façon automatique et inconsciente " (Sperber, 2000, 121). Les processus d'inférence, dont différentes versions sont développées dans la pragmatique contemporaine, sont donc construits par le destinataire du message et tendent à traiter la pertinence des informations fournies par le locuteur en se basant sur leur interprétation dans un contexte de communication particulier.

\section{Le thésaurus, un acte de discours}

32 Dès son inscription dans un thésaurus, le descripteur porte l'intention de vouloir-dire du concepteur et, pour l'indexeur qui utilise ce thésaurus, cette intention est manifeste. Tous les descripteurs sont précédés du performatif employer de manière explicite ou implicite, selon qu'ils ont ou pas de synonymes. Cette procédure ne laisse aucune alternative à l'indexeur sinon celle d'exécuter l'ordre du concepteur.

De plus, et ceci n'est pas mis en évidence par la pragmatique cognitive, la notion de choix des termes est significative dans les thésaurus, les descripteurs apparaissant sans aucune argumentation apparente. Dès lors, l'information (la connaissance?) sera, pour le spécialiste comme pour l'institution qui l'emploie, moins son objectif que l'objet autour duquel son projet se conçoit, se formalise et se diffuse. C'est cela qui instaure la primauté de l'énonciation (employer) sur un énoncé (descripteur) qui peut changer à la faveur d'une mise à jour. La force illocutionnaire est aussi amplifiée par le statut de spécialiste de l'énonciateur. C'est sans doute ainsi que l'organisation des connaissances s'élabore et s'explicite. Le langage documentaire a une dimension auto-anthropologique qui informe non pas sur un monde possible mais sur un monde réel, celui d'une institution, à travers la communication des représentations qu'elle se fait à propos de ce monde. Telle nous semble être l'une des raisons d'être des thésaurus. 

est foncièrement productif sur le plan informatif et elliptique sur le plan syntaxique. Mais ces carences peuvent être aisément comblées grâce à la dimension déictique des codes conventionnels (par exemple : terme associé, terme spécifique, etc.) et dont le nombre est suffisamment réduit pour être assimilé et restitué par la mémoire à long terme en un temps très bref. Le tableau 1 illustre ce qui est dit et ce qui est non-dit dans les thésaurus. Dans ce qui est dit, les codes déictiques sont entre crochets et dans ce qui est dit et non dit, le non dit est restitué en caractères italiques.

\begin{tabular}{|c|c|}
\hline Ce qui est dit & Ce qui est dit et non dit \\
\hline $\begin{array}{l}\text { [générique supérieur] } \\
\text { PROGRAMMES D'AIDE } \\
\text { [note d'application] } \\
\text { assistance financière } \\
\text { aux pays de } \\
\text { en voie AU } \\
\text { développement } \\
\text { [Terme générique] } \\
\text { ASSISTANCE } \\
\text { DÉVELOPPEMENT } \\
\text { [Non descripteur] } \\
\text { ASSISTANCE } \\
\text { INTERNATIONALE } \\
\text { [Termes SPécifiques] } \\
\text { AIDE BILATÉRALE } \\
\text { AIDE } \\
\text { MULTILATÉRALE } \\
\text { [Termes asSOciés] } \\
\text { ASSISTANCE } \\
\text { ÉCONOMIQUE } \\
\text { COOPÉRATION } \\
\text { TECHNIQUE }\end{array}$ & $\begin{array}{l}\text { Je (concepteur du thésaurus, spécialiste en mon domaine et employé aux } \\
\text { Nations-Unies) dis que le descripteur ASSISTANCE AU DÉVELOPPEMENT } \\
\text { représente un concept générique par rapport à AIDE BILATÉRALE et à AIDE } \\
\text { MULTILATÉRALE mais qu'il est spécifique par rapport à PROGRAMMES } \\
\text { D'AIDE. Que ce concept relève de la thématique de la THÉORIE ÉCONOMIQUE } \\
\text { du point de vue DÉVELOPPEMENT ÉCONOMIQUE et FINANCEMENT DU } \\
\text { DÉVELOPPEMENT. Ce descripteur est l'équivalent de ASSISTANCE } \\
\text { INTERNATIONALE, qui ne doit pas être employé car il n'a pas été choisi } \\
\text { comme descripteur, et il entretient une analogie de signification avec les } \\
\text { concepts ASSISTANCE ÉCONOMIQUE et COOPÉRATION TECHNIQUE. Il doit } \\
\text { être employé dans le sens de "assistance financière aux pays en voie de } \\
\text { développement". }\end{array}$ \\
\hline
\end{tabular}

Tableau 1 : Le dit et le non-dit dans les thésaurus

La combinaison du non-dit avec le descripteur, stimulée par l'insight, est perçue par le système périphérique et rapidement transférée dans la mémoire à long terme. Cette économie de temps est proportionnelle à l'économie d'énergie au niveau de l'activité cognitive de l'indexeur pour interpréter le message du concepteur/énonciateur du thésaurus.

Le je de l'énonciateur se trouve dans l'environnement institutionnel, mais par un curieux effet d'amorçage cognitif en boucle, c'est l'institutionnalisation de ce je qui lui confère une force illocutionnaire. Le non-dit se situe dans la marge entre le performatif servant à faire accomplir un acte (le fait de dire à un indexeur « employer » tel terme) et l'acte que lui-même accomplit (l'emploi effectif de ce terme par un indexeur). La dimension syntagmatique du thésaurus contient des marques discursives dont l'objectif est 
simplement de communiquer des descripteurs, au moyen du « dire » du spécialiste, pour en quelque sorte les «faire-dire » par l'indexeur et, au-delà de ce dernier, par l'usager.

Ces marques discursives absorbées par le curieux phénomène de leur silence confèrent paradoxalement davantage de vigueur au charisme de l'énonciateur. Il convient aussi de leur ajouter l'établissement de la relation d'équivalence entre termes synonymes et la note d'application qui peut receler des marques conversationnelles. Contrairement à l'approche piercienne qui considère le descripteur comme un signe ayant des occurrences dans le corpus à indexer (Le Guern, 1984; Amar, 2000), il nous semble plus approprié de l'appréhender selon l'approche austinienne, en tant qu'énoncé, prêt à l'emploi, émanant d'une instance. En cela nous rejoignons encore Austin (1970) pour qui tout usage du langage a valeur d'acte de discours.

Enfin, parmi les éléments qui peuvent rendre les choses explicites, c'est la perception d'un seul et unique élément, le descripteur, qui est susceptible de remplir une fonction bien déterminée. A partir de là, toutes les jointures entre codes et inférences, et parfois aussi les inter-inférences, semblent permises à travers un réseau combinatoire mettant en relation des milliers de descripteurs.

\section{Coopération, pertinence et connivence}

\section{Le principe de coopération}

Grice (1979) pose le principe de coopération autour d'un but commun lors de la participation des individus à un échange conversationnel. Ce but est une loi fondamentale représenté par les quatre maximes suivantes:

- la relation (talk about) concerne le but de l'échange d'information et exige que toute contribution verbale soit telle qu'elle puisse contribuer à la pertinence du discours par rapport au contexte de la conversation ;

- la quantité concerne l'efficacité de l'échange et exige que toute contribution verbale comporte un nombre suffisant et minimal d'informations ;

- la qualité vise la persuasion et exige que chaque intervenant n'affirme que ce qu'il croit être vrai ou ce de quoi il a des preuves ;

- la manière concerne la façon dont on doit dire ce que l'on dit et exige que le message soit clair, non ambigu, bref et méthodique.

Le principe de coopération se concrétise aussi dans le processus de traitement des énoncés sur la base des implications conversationnelles que nous n'aborderons pas car elles ne se prêtent pas à notre sujet.

\section{Le principe de pertinence}

Considérant que la cognition humaine vise à améliorer la connaissance que l'individu a du monde, Sperber et Wilson (1979) suggèrent la réduction des quatre maximes de Grice au seul principe de pertinence. De leur point de vue, cette amélioration peut se réaliser au moyen d'un calcul dont les prémisses, considérées comme vraies par l'auditeur, sont fournies par le savoir partagé, l'énoncé et, le cas échéant, l'énonciation. 
42 Par ailleurs, dans une interrelation humaine, quand l'intention informative est manifeste, les intérêts de l'émetteur et du récepteur sont pris en compte à un niveau présumé de pertinence qui se résume en deux principes :

Le principe cognitif de pertinence selon lequel la cognition humaine tend à maximiser la pertinence des informations qu'elle traite [...] et le principe communicationnel de pertinence selon lequel tout acte de communication communique une présomption de sa propre pertinence (Sperber et Wilson, 1989, 260).

Ce processus permet aux sources possibles des assomptions contextuelles de construire l'environnement cognitif de l'individu, qui devrait être capable d'accepter cette représentation comme vraie ou probablement vraie pour que les faits lui deviennent manifestes.

\section{Thésaurus, pertinence et connivence}

44 La notion de pertinence est particulière en science de l'information. Elle est définie comme la correspondance existant entre un document et une recherche d'information et elle est mesurable par les taux de rappel et de précision. Autrement dit, elle revient à l'évaluation du processus de l'indexation et cela ne peut se faire qu'à l'aune de l'usager. Cette notion se démarque ainsi nettement des explications de Grice (1979) et de Sperber et Wilson (1979) car elle fait intervenir le concepteur du thésaurus, l'indexeur et l'informatiste qui est rarement l'indexeur lui-même, tous trois rémunérés par l'institution, les auteurs des documents acquis par la même institution et les usagers, internes ou externes, de son système d'information. Le thésaurus se présente alors comme un langage unificateur au sein d'une interaction codique complex e où les « interinférences » joueront un rôle déterminant.

Nous nous limitons à l'activité de l'indexeur qui ne va pas consulter un thésaurus de but en blanc. Il a auparavant analysé le contenu d'un document afin d'en identifier les éléments informatifs et de reconnaître les concepts pour les exprimer sous forme de mots-clés. Ensuite, il va rechercher les descripteurs qui leur correspondent tout en les projetant sur les requêtes potentielles des usagers afin d'estimer leur charge informationnelle à la lumière des contenus disponibles. L'indexeur doit donc d'abord assimiler les codes et inférences des auteurs et les croiser avec les codes et inférences des spécialistes. Les codes des spécialistes vont logiquement s'imposer en intégrant les codes et les inférences des auteurs des documents. Sur cette base, l'indexation n'est pas concevable en tant que vulgarisation, mais plutôt en tant que restitution de ses codes au spécialiste qui les a conçus avec une plus-value informationnelle.

Certaines constructions, comme celles que nous donnons ici en exemple, incitent à approfondir davantage cette question :

Terme générique BÂTIMENTS

Termes spécifiques CENTRALES NUCLÉAIRES

CENTRES DE DOCUMENTATION PÉDAGOGIQUES

Ou encore

USAGE DE STUPÉFIANTS

[employer] ABUS DE STUPÉFIANTS

Ou enfin les notes d'application suivantes:

MANDAT 
NA : Mission confiée à un organe, accompagnée d'une délégation de pouvoirs lui permettant d'accomplir cette mission.

MANDATS

NA : Territoires sous mandat de la SDN [Société des Nations]. d'interprétations possibles. En définitive, ce double contrôle du concepteur du thésaurus sur les mots et sur le sens de son propre discours semble répondre à un objectif qui vise, à travers la bi-univocité du descripteur, la bi-directionnalité de sa perception et de sa réception en tant que représentation institutionnelle, non pas d'un concept, mais de ce qu'est un concept pour cette institution. Les prémisses, dont la majeure est que l'énonciateur est un spécialiste et la mineure est qu'il dit donc la vérité, font porter par les descripteurs la garantie de leur propre pertinence. La gestion des modules et des sousmodules est telle que le processus inférentiel de l'indexeur est obstrué, afin de ne pas interférer avec celui du concepteur. Les effets de cette gestion, notamment l'insight, et la saturation due aux milliers de descripteurs à parcourir, découvre un montage modulaire pragmatique, où la compétence de l'indexeur ne se confirme que lorsqu'il entre en parfaite connivence avec le concepteur. Des deux principes du niveau présumé de pertinence avancés par Sperber et Wilson (1979), nous n'avons retenu que le second, et nous le subordonnons à un principe de connivence, qui prime aussi sur le principe de la coopération gricéen. La pertinence prendra toute sa dimension dans un espace particulier de l'institution et avec plusieurs acteurs qui joueront tour à tour les rôles de locuteur et d'interlocuteur.

\section{Conclusion}

51 L'application de la pragmatique cognitive à l'étude des thésaurus a permis de poser les jalons d'une pragmatique de la connivence spécifique à ce type de langage. L'objectif étant de communiquer une organisation de la connaissance, élaborée par un spécialiste en son domaine, à un indexeur du savoir, la modularité et le phénomène de l'insight sont exploités par le concepteur dans le but de fournir rapidement au destinataire à la fois le code et l'inférence qui lui est associée par le biais des énonciations performatives et de la force illocutionnaire. Il s'avère que la modularité et le non-dit discursif, mentalement restituable par le destinataire, sont motivés par le principe d'économie qui sous-tend les deux processus de traitement et de communication de l'information. Les intentions manifestes, la conscience partagée de l'environnement, du statut du concepteur, et de la fonction de l'indexeur constituent des prémisses tenues pour vraies et contribuent à la pertinence des descripteurs. Enfin, l'absence d'argumentation quant au choix des descripteurs fonctionne en elle-même comme un argument en faveur de sa bi-univocité et de sa pertinence. Tous ces éléments constituent les bases d'un principe de connivence, entre énonciateur des descripteurs et indexeur, qui apparaît comme la condition de la réception des messages et de la réalisation de l'acte d'indexer. 


\section{BIBLIOGRAPHIE}

Amar M., (2000), Les fondements théoriques de l'indexation : une approche linguistique, Paris, ADBS.

Austin J. L., (1970), Quand dire, c'est faire, Paris, Seuil (Trad. de How to do things with Words, Oxford, Urmson, 1962).

Fodor J. A., (1986), La modularité de l'esprit : essais sur la psychologie des facultés, Paris, Minuit. Coll. Propositions.

Grice H. P., (1957), Meaning, The Philosophical Review, vol. 66, n 3, pp. 377-388.

Grice H. P., (1979), Logique et conversation, Communications, $n^{\circ} 30$, pp. 57-72.

Le Guern M., (1984), Les descripteurs d'un système documentaire : essai de définition, Condenser, suppl. 1, pp. 163-169.

Sperber D., (2000), La communication et le sens, Qu'est-ce que l'humain ?, Université de tous les savoirs, vol. 2, Paris, Odile Jacob, pp. 119-128.

Sperber D. et Origi G., (2005), Pourquoi parler, comment comprendre ?, Jean-Marie Hombert (éd.), L'origine de l'homme, du langage et des langues, Paris, Fayard, pp. 236-253.

Sperber D. et Wilson D., (1979), Remarques sur l'interprétation des énoncés selon Paul Grice, Communications, $\mathrm{n}^{\circ} 30$, pp. 80-94.

Sperber D. et Wilson D., (1989), La pertinence : communication et cognition, Paris, Minuit.

\section{NOTES}

1. Tous les exemples sont issus du Thésaurus du Système d'Information Bibliographique des Nations Unies, édition de 1986.

\section{RÉSUMÉS}

L'adaptation de la pragmatique cognitive à l'étude de l'organisation des connaissances dans les thésaurus a mis à l'épreuve la théorie des actes de langage et la théorie de l'esprit. La description de la modularité des thésaurus démontre que le concepteur transmet à la fois le code linguistique et l'inférence qui lui est associée, et met en évidence l'effet de l'insight sur l'indexeur. De plus, le thésaurus comporte des énonciations performatives et recèle des marques discursives distinctives des actes de langage. Sur cette base, dans le cadre de la communication interindividuelle, le principe de pertinence de la pragmatique cognitive, issu des maximes du principe de coopération gricéen, a été revisité et récupéré sous un principe de connivence dont les 
conditions essentielles sont la conscience partagée de l'environnement et des statuts respectifs de chaque acteur concerné par les thésaurus.

The adaptation of cognitive pragmatics to the study of the organization of knowledge in a thesaurus has put to the test both the theory of speech acts and the theory of mind. The description of the modularity of a thesaurus shows that thesaurus designers transmit both the linguistic code and its associated inferences and highlight the effect of insights on the indexer. The thesaurus also contains performative utterances as well as discursive marks, which are distinct from speech acts. On this basis and within the framework of inter-personal communication, the principle of relevance described in cognitive pragmatics and derived from the Gricean cooperative principle is revisited and re-used as a principle of "connivance," whose essential conditions are a shared awareness of the environment and of the respective status of each actor involved in the thesaurus.

\section{INDEX}

Keywords : thesaurus, cognitive pragmatics, modularity of language, speech acts, principle of connivance

Mots-clés : thésaurus, pragmatique cognitive, modularité du langage, actes de langage, principe de connivence

\section{AUTEUR}

\section{EL-KHANSA MKADA}

El-Khansa Mkada, Institut supérieur de documentation, Université de la Manouba - Tunisie 